\title{
Influence of the pore generation method on the metal dispersion and oxidation activity of supported Pt in monolithic catalysts
}

\author{
R. Portela ${ }^{1}$, V.E. García-Sánchez ${ }^{1}$, M. Villarroel ${ }^{2}$, S.B. Rasmussen ${ }^{1}$, and P. Ávila ${ }^{1 *}$ \\ ${ }^{1}$ Institute of Catalysis and Petrochemistry-CSIC, Madrid (Spain) \\ ${ }^{2}$ Fac. de Química y Biología, USACH, Santiago de Chile (Chile) \\ pavila@icp.csic.es
}

\begin{abstract}
The catalytic oxidation activity of structured macro/mesoporous Pt catalysts obtained by the one-step impregnated carbon procedure (ICP) with different binder/support combinations (sepiolite or mixtures with $\mathrm{TiO}_{2}$ or $\mathrm{Al}_{2} \mathrm{O}_{3}$ ) is compared to that of catalysts with the same composition obtained by conventional impregnation of the previously shaped support, with and without activated carbon as pore-generating agent. The superior results of the former are explained in terms of porosity and metal dispersion, estimated by calculation of the platinum apparent molar surface coverage from electrophoretic migration measurements. The big macropores generated reduce the internal diffusion limitations, facilitating the accessibility of the gaseous compounds to the active phase. However, the main improvement for these sepiolite-based monoliths must be mainly attributed to the good Pt dispersion obtained by this method, with metal particles smaller than $10 \mathrm{~nm}$, confirming the role of the activated carbon template not only as a macroporosity generating agent, but also as a dispersant and stabilizer of the metal particles -preventing agglomeration-, and as source of $\mathrm{CO}$ for in situ reduction of the precursor. In addition, the ICP method minimizes the calcination steps needed for the preparation of the catalyst.
\end{abstract}

Key words: VOCs; oxidation; dispersion; porosity; monolith

\section{INTRODUCTION}

Supported noble metals are widely used in the industry, and their activity as well as their economic viability depends on the metal dispersion on the surface of the support and the accessibility of the active phase. To optimize these parameters while minimizing the environmental impact and economical costs of the catalysts is a priority for the society and the industry. The conventional preparation methods for these types of catalysts are relatively complex and costly, since they involve several synthesis stages [1]:

a) preparation of a high surface area support,

b) incorporation of the active phase precursor, generally by wet impregnation or chemical vapor deposition, and

c) reduction of the metal precursor to obtain the active metallic phase.

Therefore, a series of heat treatments are usually needed to obtain the final catalyst:

a) calcination of the support in the adequate conditions (temperature and atmosphere) so as to obtain the desired phase transformations and eliminate any hard template used as pore generating agent (PGA) or other additives; 
b) drying to remove the solvent of the precursors solution, during this process the dissolved salts may migrate from inside the pores to the entrance, resulting in poor dispersion of the active phase;

c) calcination at high temperatures $\left(300-600^{\circ} \mathrm{C}\right)$ in a reducing atmosphere to convert the precursors into active phases, a process usually accompanied by salt decomposition reactions with the consequent release of gases.

The drying stage and the final heat treatment are challenging. They can induce agglomeration or sintering of the active phases, resulting in low dispersion, with the subsequent decrease in the catalytic performance. In environmental and industrial applications catalytic supports are often shaped as monolithic structures with parallel channels because it gives the superior balance between low pressure drop in the reactor and high geometric surface area of the catalytic material [2]. Also the specific surface area, related to smaller pores, must be maximized in order to increase the number of available active sites, and the wall thickness must be minimized to reduce catalyst volume and internal diffusion limitations. Moreover, meso and macroporosity may facilitate the accessibility of active sites located within the walls with a less significant decrease of mechanical strength compared to a further reduction of wall thickness [3].

The literature reports on the generation of meso and/or macroporous materials during the synthesis process using surfactants as soft templates [4] or hard templates such as polymers [5] or carbonaceous materials [6]. In the last years the preparation of ceramic structured supports with high and hierarchical porosity has gathered the attention of the scientific community [3]. Highly porous magnesia, with macropores in the micron range and mesopores smaller than $100 \mathrm{~nm}$, has been obtained by spray freeze drying of magnesium sulfate solutions, where fine salt particles with open pores were produced due to sublimation of ice crystal, after calcination at temperatures up to $1300-1500{ }^{\circ} \mathrm{C}$ [7]. $\mathrm{Si}-\mathrm{B}-\mathrm{C}-\mathrm{N}$ monoliths with high, interconnected and hierarchical meso and macroporosity have been prepared by spark plasma sintering of ordered mesoporous powders at 800 $1000{ }^{\circ} \mathrm{C}$ [8]. Oxidation-bonding of graphite incorporated into $\mathrm{SiC}$ powders yields high porosity ceramics with tunable pore diameter [9]. Moreover, hierarchical macromesoporous monoliths can be also prepared combining sol-gel routes with phase separation to obtain $\mathrm{TiO}_{2}$ [10], mullite [11] or cordierite [12].

Earlier some of us prepared sepiolite and titania-sepiolite honeycomb monoliths from commercial powders by extrusion using activated carbon as template for macroporosity generation [13]. The addition of activated carbon resulted in the enlargement of the interparticular distances, without chemical interaction with the other components of the support. More recently we proposed the so-called "impregnated carbon procedure" (ICP) [14], which gives very active functionalized structured materials minimizing energy and reagents consumption by in situ reduction and dispersion of the metal precursor together with the generation of porosity in one single calcination step [15]. The method consists in the kneading of an aqueous paste composed by a binder and activated carbon (AC) particles, where the active phase precursor has been pre-impregnated. Additionally, a metal oxide can be included as support. After extrusion of the paste, the green body presents the desired final form of the catalyst: honeycomb, pellet, etc. This material is then transformed in one step into an activated dispersed catalyst by heat treatment in a lean oxygen atmosphere. The calcination conditions facilitate the burnout of the carbon particles, whereby macroporosity is generated simultaneously with an intra-pore gasphase transfer of the metal to the final support in a similar way to chemical vapor 
deposition. The activated carbon dispersed throughout the material serves as a reducing element of metallic cations either directly or by the action of carbon monoxide formed at the sites of combustion with low oxygen supply. Thus, shaped catalysts can be directly prepared in their final monolithic form in one step, adding a green chemistry value [16] to the synthesis process.

The ICP method has been optimized regarding activated carbon particle size and amount, heat treatment temperature, and particle size and loading of platinum [17]. However, the higher performance of catalysts prepared with impregnated carbon with respect to those obtained by conventional impregnation can be related to several factors and their role has not been assessed yet. In order to get a deeper understanding, here the ICP method is employed to synthesize structured macro/mesoporous $\mathrm{Pt}$ catalysts with different binder/support combinations (only sepiolite or mixtures with $\mathrm{TiO}_{2}$ or $\mathrm{Al}_{2} \mathrm{O}_{3}$ ). The catalytic oxidation activity of the samples obtained by the ICP method is compared to that of catalysts with the same composition obtained by conventional impregnation of the previously shaped support, with and without activated carbon as pore-generating agent. The role of porosity and metal dispersion in the catalytic activity is investigated as a function of the synthesis method and the properties of the support.

\section{EXPERIMENTAL}

Three series of similar catalysts in the shape of pellets were prepared with sepiolite as binder (Pansil 100, Tolsa S.A.): in one series sepiolite is binder and support for the noble metal, whereas in the other two series $50 \mathrm{w} \%$ of a metal oxide is added as support, either alumina (Pural SB, Condea) or titania (G5, Millenium). Three different methods were employed for each series of supports to obtain monoliths with $0.2 \mathrm{wt} \% \mathrm{Pt}$ using chloroplatinic acid as precursor: i) conventional synthesis of the monolithic support by extrusion and calcination at $550^{\circ} \mathrm{C}$ (samples referred to as $\mathrm{Sep}, \mathrm{SepAl}_{2} \mathrm{O}_{3}$, or $\mathrm{SepTiO}_{2}$, depending on the support), followed by impregnation and further heat-treatment in $\mathrm{NH}_{3} / \mathrm{N}_{2}$ (samples referred to as Pt-Support (Imp)), as described in [18]; ii) impregnated carbon procedure $[14,15]$, where the calcination steps are reduced from two to one at $550^{\circ} \mathrm{C}$ by adding pre-impregnated activated carbon (Fluesorb B, Chemviron) to the dough for both porosity generation and metal dispersion (samples referred to as Pt-Support (ICP)); and iii) two-step process similar to $i$, but with pore generation by incorporation of nonimpregnated activated carbon during the synthesis of the support (same type and quantity as in method ii, samples referred to as Support (PGA) and Pt-Support (PGA) before and after impregnation) [19].

The textural properties of the materials were determined using the complementary information obtained from nitrogen sorption isotherm and mercury intrusion porosimetry (MIP). Data between $7 \mathrm{~nm}$ and $300 \mu \mathrm{m}$ were obtained using a CE Instruments Pascal $140 / 240$ mercury intrusion porosimeter. The samples were dried overnight at $150{ }^{\circ} \mathrm{C}$. The pore diameters were calculated by the Washburn equation [20], assuming a nonintersecting cylindrical pore model, using the values recommended by the IUPAC for the mercury contact angle $\left(141^{\circ}\right)$ and surface tension $\left(484 \mathrm{mN} \mathrm{m}^{-1}\right)$ [21]. Data related to micropores and mesopores $<7 \mathrm{~nm}$ were calculated from nitrogen sorption isotherms at $196{ }^{\circ} \mathrm{C}$ determined on either a Carlo Erba Sorptomatic 1800 or a Micromeritics ASAP 2420. The samples were previously outgassed overnight at $300{ }^{\circ} \mathrm{C}$ to a vacuum of less than $1.33 \times 10^{-2} \mathrm{~Pa}$ to ensure a dry clean surface, free from any loosely bound adsorbed species. Pore size distribution and specific surface area was calculated by application of the Barrett-Joyner-Halenda (BJH) model [22], based on Kelvin equation and Brunauer- 
Emmett-Teller (BET) adsorption theory, to the desorption branch taking the area of the nitrogen molecule as $0.162 \mathrm{~nm}^{2}$ [21].

Electrophoretic migration measurements were carried out on a Zeta Meter 3.0 using a suspension containing $30 \mathrm{mg}$ of sample in $300 \mathrm{~mL}$ of a $10^{-3} \mathrm{M} \mathrm{KCl}$ solution [23]. $\mathrm{pH}$ was adjusted with $0.1 \mathrm{M} \mathrm{HCl}$ or $\mathrm{NaOH}$ to obtain a proper set of zeta potential point, calculated using the Helmholtz-Smoluchowski equation, that allow the determination of the zero point charge (ZPC).

The morphology of the particles was observed in a JEOL $2100 \mathrm{~F}$ transmission electron microscope (TEM). For the analysis, a drop of a solution containing the grinded extrudates was deposited on the sample grid.

Phase distribution in representative areas of the catalysts was determined by wavelengthdispersive spectroscopy - electron probe microanalysis (WDS-EPMA), using a JEOL JXA-8900M apparatus with a resolution power of $6 \mathrm{~nm}$ and a voltage of $20.0 \mathrm{kV}$. Crosssections of the samples about $5 \mathrm{~mm}$ thick were embedded in resin, polished with diamond to avoid surface irregularities that may distort the analysis, and coated with a film of graphite to increase the electrical conductivity.

The catalytic activity was evaluated performing the combustion of volatile organic compounds (VOCs) as model reaction test. The experiments were conducted in a glass tubular reactor that operates in integral regime placed in the axis of a PID-controlled oven. Inlet and outlet total organic carbon (TOC) concentration was measured with a Beckman Industrial Model 400A flame ionization detector (FID). The catalyst, grinded to obtain particles with $\phi=1-2 \mathrm{~mm}$, was placed in the central part of the reactor with the help of glass wool plugs. The reaction temperature varied between 0 and $200{ }^{\circ} \mathrm{C}$. The air flow rate was fixed to $2 \mathrm{~L} \mathrm{~min}^{-1}$ by means of mass flow controllers. Part of the air stream $\left(20 \mathrm{~mL} \mathrm{~min}^{-1}\right)$ was bubbled through a thermostated saturator at $50{ }^{\circ} \mathrm{C}$ containing a saturated formalin solution stabilized with methanol (formaldehyde/methanol/water = $37 / 15 / 48 \mathrm{w} \%$ ) to obtain an inlet TOC concentration of $200 \mathrm{ppmv}$. The main operating conditions are collected in Table 1.

Table 1. Catalytic tests operating conditions

\begin{tabular}{lll}
\hline Flow rate, F & 1.9 & $\mathrm{~L} \mathrm{~min}^{-1}$ \\
[TOC $_{0}$ & 200 & $\mathrm{ppmv}$ \\
Bed height & 45 & $\mathrm{~mm}$ \\
Reactor inner diameter & 11.6 & $\mathrm{~mm}$ \\
Particle diameter & $1-2$ & $\mathrm{~mm}$ \\
Catalyst volume, V & 4.8 & $\mathrm{~mL}$ \\
GHSV & 24000 & $\mathrm{~h}^{-1}$ \\
Lineal velocity & 0.3 & $\mathrm{~m} \mathrm{~s}^{-1}$ \\
V/F & 0.15 & $\mathrm{~s}$ \\
\hline
\end{tabular}




\section{RESULTS AND DISCUSSION}

\subsection{Textural properties}

Natural sepiolite (magnesium silicate, Pansil 100, Tolsa) has been used as binder for the preparation of the honeycomb monoliths. The main role of sepiolite when titania or alumina are used as supports, in agreement with our previous experience [24, 25], is to improve the rheological properties of the ceramic paste during the extrusion process and to provide the heat-treated composite with mesoporosity and mechanical strength. No significant chemical interaction of sepiolite with the support was detected, and the addition of sepiolite basically improved the textural properties of the material. Figure 1 displays the pore volume of the shaped supports as a function of pore size, both as cumulative values and as differential distribution, obtained by combination of $\mathrm{N}_{2}$ isotherms and MIP. All nitrogen adsorption-desorption isotherms were of type II with H3 hysteresis loops typical for materials with ill-defined mesoporosity that extends into the macropore range. It is well known that the micropores of natural sepiolite [26] collapse during the thermal treatment at $550{ }^{\circ} \mathrm{C}$ and the structure folds into the anhydrous form [27]; as a result the pore size distribution of the sepiolite support shown in Figure 1A corresponds to interfibrillar spaces and falls in the meso/macro porosity range $[28,29]$. The total pore volume of the support, $0.5 \mathrm{~mL} \mathrm{~g}^{-1}$, is almost triplicated with the addition of $30 \mathrm{w} \%$ of activated carbon to the dough before extrusion (ca. $0.8 \mathrm{~mL} \mathrm{~g}^{-1}$, maximum at $2-5$ $\mu \mathrm{m})$, due to the high interparticular macroporosity formed by combustion of the temporal additive. The pore size distribution and total porosity of the sepiolite supports is not significantly affected by the conventional impregnation process (Figure 1B) and the same is observed for the silicate/oxide mixed supports. 

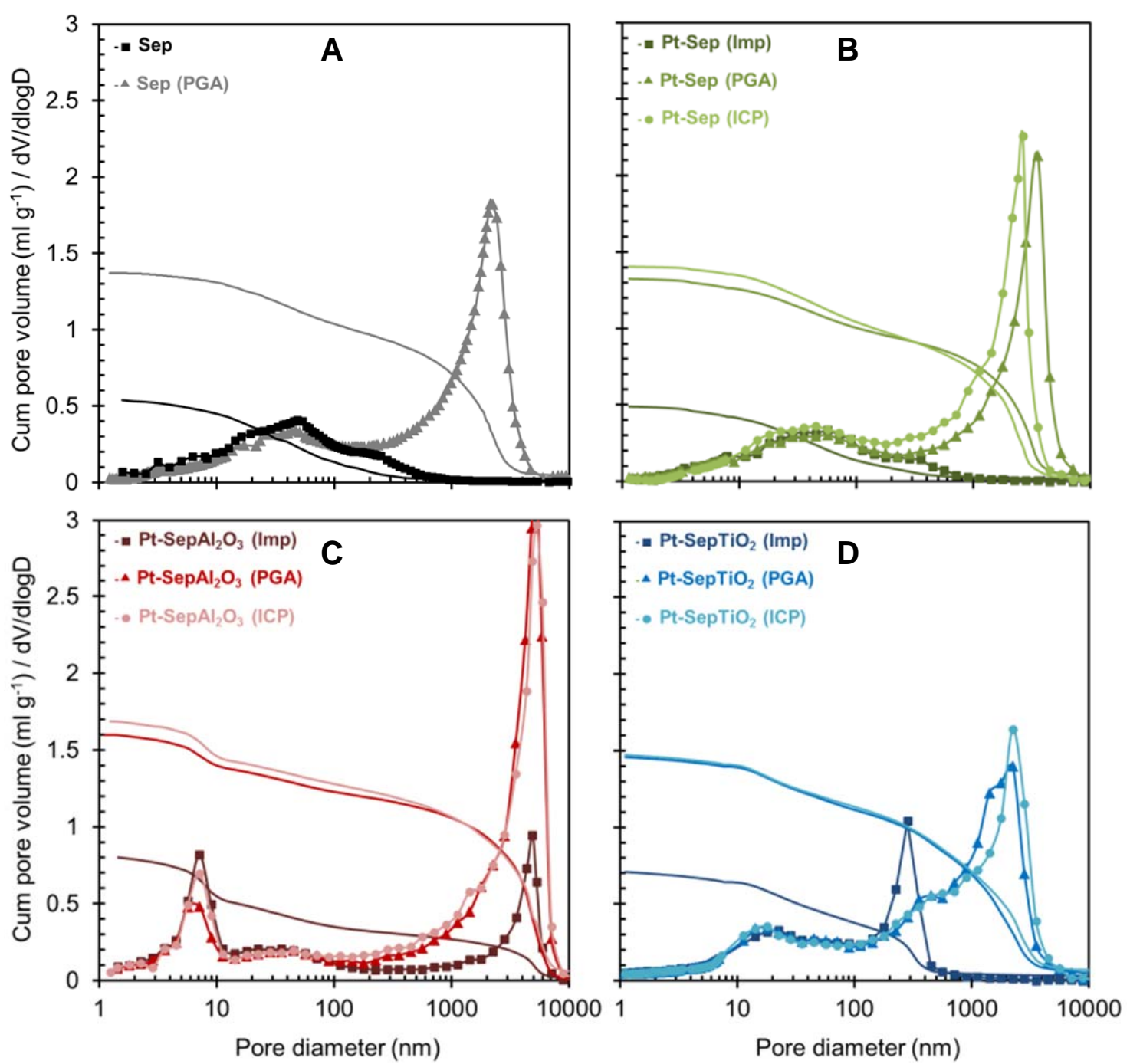

Figure 1. Pore size distribution (lines + symbols) and cumulated pore volume (lines) of sepiolite-based structured monoliths: A) Sepiolite supports, B) Pt-Sep catalysts, C) Pt-SepAl $\mathrm{O}_{3}$ catalysts, and D) Pt$\mathrm{SepTiO}_{2}$ catalysts.

Sep- $\mathrm{Al}_{2} \mathrm{O}_{3}$ and $\mathrm{Sep}-\mathrm{TiO}_{2}$ monoliths before and after impregnation are characterized by the porosity of the sepiolite component and the macroporosity associated to the oxide particles spacing, with maxima at $5 \mu \mathrm{m}$ and $280 \mathrm{~nm}$, respectively, due to the larger size of the alumina particles (Figure $1 \mathrm{C}$ and $1 \mathrm{D}$ ). Sep- $\mathrm{Al}_{2} \mathrm{O}_{3}$ monolith has additional pores smaller than $10 \mathrm{~nm}$, related to the intrinsic porosity of the alumina, and thus presents a trimodal distribution and high specific surface area. The total pore volume of the mixed monoliths prepared with activated carbon, either by PGA or ICP methods, is doubled, in a similar effect to that observed with bare sepiolite support (Figures 1A and 1B) and in previous works using other metal oxides [30]. dV/dlogD curve of Pt-Sep- $\mathrm{TiO}_{2}$ sample combines the size of macropores created by the hard template and of the original titania interparticular voids in a broad macropore size distribution $(0.3$ to $5 \mu \mathrm{m})$; meanwhile the size of macropores in Pt-Sep- $\mathrm{Al}_{2} \mathrm{O}_{3}$ is not significantly changed by the pore generating agent, just the volume. The intrinsic pores of the alumina particles explain the large BET surface area of this series of solids. Table 2 shows the textural characteristics of the shaped materials prepared, including MIP and BET surface area and the fractions of micro- meso and macro-pores, together with the total pore volume. 
Table 2. Textural properties of the monolithic catalysts and supports calcined at $550^{\circ} \mathrm{C}$.

\begin{tabular}{|c|c|c|c|c|c|c|}
\hline Sample & $\underset{\left(\mathrm{m}^{2} \mathrm{~g}^{-1}\right)}{\mathrm{S}_{\mathrm{MP}}}$ & $\begin{array}{c}\mathrm{S}_{\mathrm{BET}} \\
\left(\mathrm{m}^{2} \mathrm{~g}^{-1}\right)\end{array}$ & $\begin{array}{l}\text { Micropore } \\
\left(\mathrm{cm}^{3} \mathrm{~g}^{-1}\right)\end{array}$ & $\begin{array}{l}\text { Mesopore } \\
\left(\mathrm{cm}^{3} \mathrm{~g}^{-1}\right)\end{array}$ & $\begin{array}{l}\text { Macropore } \\
\left(\mathrm{cm}^{3} \mathrm{~g}^{-1}\right)\end{array}$ & $\begin{array}{l}\text { Total pore V } \\
\left(\mathrm{cm}^{3} \mathrm{~g}^{-1}\right)\end{array}$ \\
\hline Sep & 87.0 & 135.7 & 0.18 & 0.34 & 0.03 & 0.55 \\
\hline Sep (PGA) & 53.5 & 116.8 & 0.14 & 0.38 & 0.85 & 1.37 \\
\hline Pt-Sep (Imp) & 52.4 & 113.0 & 0.16 & 0.30 & 0.03 & 0.49 \\
\hline Pt-Sep (PGA) & 49.8 & 115.5 & 0.14 & 0.33 & 0.86 & 1.32 \\
\hline Pt-Sep (ICP) & 59.6 & 112.1 & 0.15 & 0.43 & 0.83 & 1.40 \\
\hline $\mathrm{SepAl}_{2} \mathrm{O}_{3}$ & 137.4 & 189.4 & 0.32 & 0.18 & 0.29 & 0.79 \\
\hline $\mathrm{SepAl}_{2} \mathrm{O}_{3}(\mathrm{PGA})$ & 141.5 & 170.6 & 0.30 & 0.24 & 1.18 & 1.72 \\
\hline Pt-SepAl $\mathrm{O}_{3}(\mathrm{Imp})$ & 69.9 & 188.6 & 0.33 & 0.17 & 0.30 & 0.80 \\
\hline $\mathrm{Pt}-\mathrm{SepAl}{ }_{2} \mathrm{O}_{3}(\mathrm{PGA})$ & 50.2 & 188.9 & 0.26 & 0.20 & 1.14 & 1.60 \\
\hline Pt-SepAl ${ }_{2} \mathrm{O}_{3}(\mathrm{ICP})$ & 63.5 & 182.5 & 0.29 & 0.25 & 1.14 & 1.69 \\
\hline $\mathrm{SepTiO}_{2}$ & 88.0 & 107.5 & 0.14 & 0.54 & 0.03 & 0.71 \\
\hline $\mathrm{SepTiO}_{2}(\mathrm{PGA})$ & 81.2 & 100.8 & 0.13 & 0.52 & 0.80 & 1.45 \\
\hline $\mathrm{Pt}_{-\mathrm{SepTiO}}(\mathrm{Imp})$ & 52.1 & 106.4 & 0.16 & 0.50 & 0.06 & 0.71 \\
\hline Pt-SepTiO $2(\mathrm{PGA})$ & 58.7 & 98.2 & 0.17 & 0.45 & 0.84 & 1.46 \\
\hline Pt-SepTiO ${ }_{2}(\mathrm{ICP})$ & 60.1 & 98.8 & 0.18 & 0.44 & 0.86 & 1.47 \\
\hline
\end{tabular}

There is a parallelism between the surface area values obtained by MIP and BET, though MIP values are lower because mercury cannot intrude in pores smaller than $5 \mathrm{~nm}$, which represent a negligible fraction of the total pore volume but significantly contribute to the surface area of the solid. Apparently in the process of macroporosity generation with AC some pores become interconected, slightly reducing the BET surface area of the supports. More significantly, MIP surface area is much lower for the samples containing Pt, particularly in the solids containing alumina, while BET surface area is not significantly reduced, suggesting that the active phase is mainly occupying the external surface area, including the small mesopores of the alumina particles.

\subsection{Catalytic activity}

For each series of catalysts, with fixed composition, the catalytic performance of the samples depends significantly on the preparation method. Figure 2 displays the catalytic activity of the three series for $\mathrm{HCHO}-\mathrm{CH}_{3} \mathrm{OH}$ mixture oxidation, expressed as total VOC conversion vs temperature. The addition of a metal oxide support to sepiolite is detrimental for the catalytic activity of the solids prepared by impregnation of a shaped 
support: among Imp-samples Pt-Sep (Imp) displays the best performance. The impregnation of the silicate/oxide mixed supports with increased macro-porosity leads to more efficient catalysts than $\mathrm{Pt}_{-} \mathrm{SepAl}_{2} \mathrm{O}_{3}$ (Imp) and $\mathrm{Pt}-\mathrm{SepTiO}_{2}$ (Imp), which is represented by a higher slope in the activity curves over temperature, observed in Figures $2 \mathrm{~B}$ and $2 \mathrm{C}$. The benefit of the generation of macroporosity in the mixed supports is attributed to the reduction of the diffusional limitations. This enhancement of the diffusional properties, however, is not translated into a better catalytic performance in PtSep (PGA) sample, which is the worst performing sample among all the catalysts prepared, with very limited conversion values even at $100{ }^{\circ} \mathrm{C}$. Moreover, the marked activity decrease with respect to Pt-Sep (Imp), and the consequent shift of the conversion curve towards lower temperature values observed in Figure 2A, indicates that the porosity generated by the PGA in the sepiolite support negatively affects the catalytic properties of the Pt incorporated by the subsequent impregnation procedure, and therefore additional parameters must be considered to explain this effect.
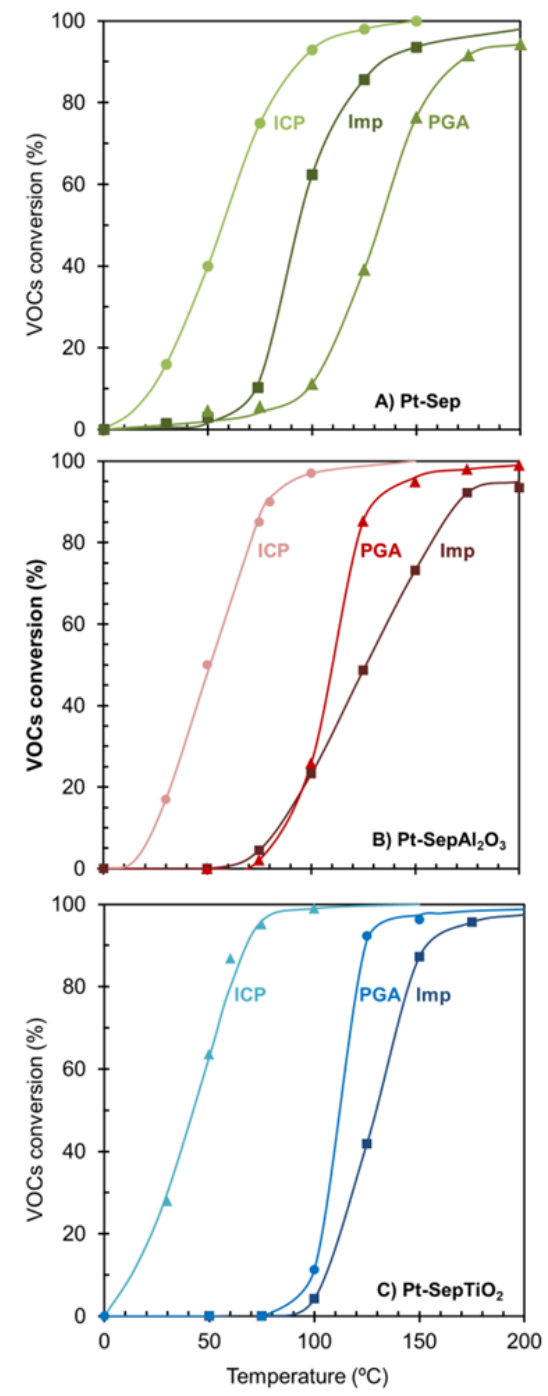

Figure 2. Catalytic oxidation performance as a function of the preparation method of the three catalytic series: A) Pt-Sep, B) Pt-Sep $\mathrm{Al}_{2} \mathrm{O}_{3}$ and C) Pt- Sep $\mathrm{TiO}_{2}$

The catalysts prepared by the impregnated carbon procedure are the most efficient. ICPsamples present catalytic activity at room temperature and achieve total VOCs elimination at $120^{\circ} \mathrm{C}$ with any kind of support, following the order Pt-SepTiO 2 (ICP)>Pt- 
$\mathrm{SepAl}_{2} \mathrm{O}_{3}(\mathrm{ICP})>\mathrm{Pt}-\mathrm{Sep}(\mathrm{ICP})$. The enhancement obtained with the ICP-samples is very significant, even if only sepiolite is used as support, which yields the best conventionally impregnated catalyst and the worst ICP-catalyst, and is equivalent to that achieved for combustion of toluene with similar catalysts [14]. Taking into consideration that the active phase precursor and amount are the same for all samples and the composition is constant in each series of catalysts, the dispersion of the active phase must be considered to explain the much higher activity of ICP-samples compared to PGA-samples and the bad performance of Pt-Sep (PGA) with respect to Pt-Sep (Imp). Metal dispersion estimations by temperature-programmed reduction with $\mathrm{H}_{2}$ and $\mathrm{CO}$ are not possible in these samples due to the low Pt content $(0.2 \%)$ and the complexity of the natural sepiolite clay, which contains many impurities that significantly interfere in the results obtained by these typically employed techniques. Thus, the distribution of platinum in the catalytic solids was assessed by estimation of the Pt surface coverage, TEM, and WDSEPMA.

\subsection{Pt surface coverage}

Taking as reference the Parks equation (Eq. 1) [31], and considering that the zero point of charge (ZPC) depends on the surface properties, Gil-Llambías proposed that the ZPC of a catalytic material is a linear combination of the ZPC of the support and the metal (oxide) acting as active phase [32]. Thus, the metal apparent molar surface coverage $\left(\mathrm{X}_{\mathrm{M}}\right)$ can be estimated by Eq. 2 using zeta potential data calculated from electrophoretic migration measurements [23].

$\mathrm{ZPC}=\Sigma \mathrm{X}_{\mathrm{i}}(\mathrm{ZPC})_{\mathrm{i}}$

[Eq. 1]

$\mathrm{X}_{\mathrm{M}}=\left(\mathrm{ZPC}-\mathrm{ZPC}_{\mathrm{S}}\right) /\left(\mathrm{ZPC}_{\mathrm{M}}-\mathrm{ZPC}_{\mathrm{S}}\right)$

Table 3 collects the Pt apparent molar surface coverage $\left(\mathrm{X}_{\mathrm{Pt}}\right)$ calculated for each material, considering the $\mathrm{ZPC}$ of $\mathrm{PtO}_{\mathrm{x}}(\mathrm{pH}=14)$ [31] and the corresponding $\mathrm{ZPC}_{\mathrm{S}}$ for each kind of support. The significant difference between the very high ZPC of the active phase and the ZPC of the supports, $\sim 2-3$, makes this method greatly sensitive to small coverage variations despite the low Pt mass ratio in the solid materials under study. 
Table 3. Pt apparent molar surface coverage

\begin{tabular}{|c|c|c|}
\hline Sample & $\begin{array}{l}\mathrm{ZPC} \\
(\mathrm{pH})\end{array}$ & $\begin{array}{c}\mathrm{X}_{\mathrm{Pt}} \\
\text { (molar) }\end{array}$ \\
\hline Sep & 2.21 & - \\
\hline Sep (PGA) & 2.45 & - \\
\hline Pt-Sep (Imp) & 2.35 & 0.012 \\
\hline Pt-Sep (PGA) & 2.47 & 0.002 \\
\hline Pt-Sep (ICP) & 3.01 & 0.048 \\
\hline $\mathrm{SepAl}_{2} \mathrm{O}_{3}$ & 2.09 & - \\
\hline $\mathrm{SepAl}_{2} \mathrm{O}_{3}(\mathrm{PGA})$ & 2.30 & - \\
\hline Pt-SepAl ${ }_{2} \mathrm{O}_{3}(\mathrm{Imp})$ & 2.55 & 0.039 \\
\hline Pt-SepAl ${ }_{2} \mathrm{O}_{3}(\mathrm{PGA})$ & 2.82 & 0.044 \\
\hline Pt-SepAl $\mathrm{O}_{3}(\mathrm{ICP})$ & 2.95 & 0.056 \\
\hline$\overline{\mathrm{SepTiO}_{2}}$ & 2.45 & - \\
\hline $\mathrm{SepTiO}_{2}(\mathrm{PGA})$ & 2.48 & - \\
\hline $\mathrm{Pt}_{-S e p T i O}(\operatorname{Imp})$ & 2.49 & 0.003 \\
\hline Pt-SepTiO $2(\mathrm{PGA})$ & 2.53 & 0.004 \\
\hline $\mathrm{Pt}-\mathrm{SepTiO}_{2}(\mathrm{ICP})$ & 2.76 & 0.024 \\
\hline
\end{tabular}

$\mathrm{X}_{\mathrm{Pt}}$ values at equal Pt loading, representative of the active phase dispersion, correlate well with the bad performance of Pt-Sep (PGA) and the activity enhancement obtained with the ICP method in all samples, and can be explained by the fact that during the impregnation of solid supports with active phase precursors the ZPC of the support, the $\mathrm{pH}$ of the solution, and the nature and concentration of the precursor ionic species play a key role in the interaction precursor/support, and thus in the final dispersion obtained [33]. The $\mathrm{pH}$ of the chloroplatinic acid solution used for the impregnation of the catalysts is ca. 4-5 and $\mathrm{Pt}$ is in anionic form, either as $\left[\mathrm{PtCl}_{6}\right]^{2-}$ or $\left[\mathrm{PtCl}_{5} \mathrm{H}_{2} \mathrm{O}\right]^{-}$[34]. The low $\mathrm{ZPC}$ of sepiolite hinders the impregnation of the natural silicate with $\mathrm{Pt}$ at $\mathrm{pH}>2$ because chloroplatinate anions are strongly repelled by negatively charged sepiolite particles [1]. As the solvent is progressively removed from the pores during the drying process chloroplatinate anions are repelled by the pore walls and concentrate in the remaining solution, which facilitates platinum precipitation in the pores. At higher pore size the formation of bigger Pt aggregates is favored. In order to improve sepiolite impregnation a salt with platinum in the positively charged ion should be selected as precursor, such as the tetraamin nitrate or the chloride, or the $\mathrm{pH}$ of the impregnating solution should be lower than the ZPC of sepiolite [35]. However, with highly acidic doughs the extrusion machine is damaged, so the ICP method cannot be employed. The mixture of sepiolite with alumina or titania, with ZPC of 9 and 6 respectively (Figure 4), should be a good alternative as well. During impregnation alumina and titania are positively charged, which favors the deposition of the metal precursor on their surface instead of on sepiolite. 


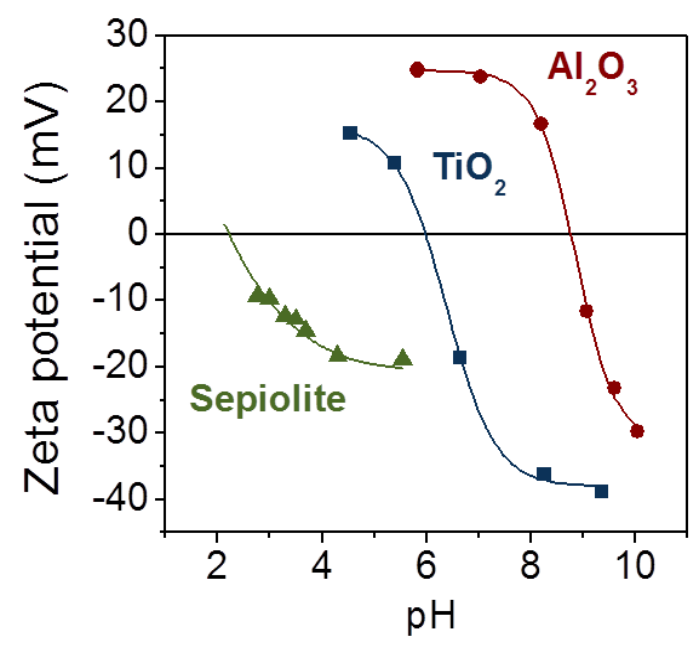

Figure 4. Zeta potential curves of the raw materials obtained by electrophoretic migration measurements.

The temperature of half VOC conversion $\left(\mathrm{T}_{50}\right)$ is plotted in Figure 5A vs surface coverage to analyze dispersion-activity relationships in these materials. For a given composition there is an exponential decay of $\mathrm{T}_{50}$, i.e. an exponential activity increase, as the Pt surface coverage increases. The higher dispersion, greatly favored in the ICP preparation method, can be correlated to a higher accessibility of the active phase for the reactant (see Figure 5B).
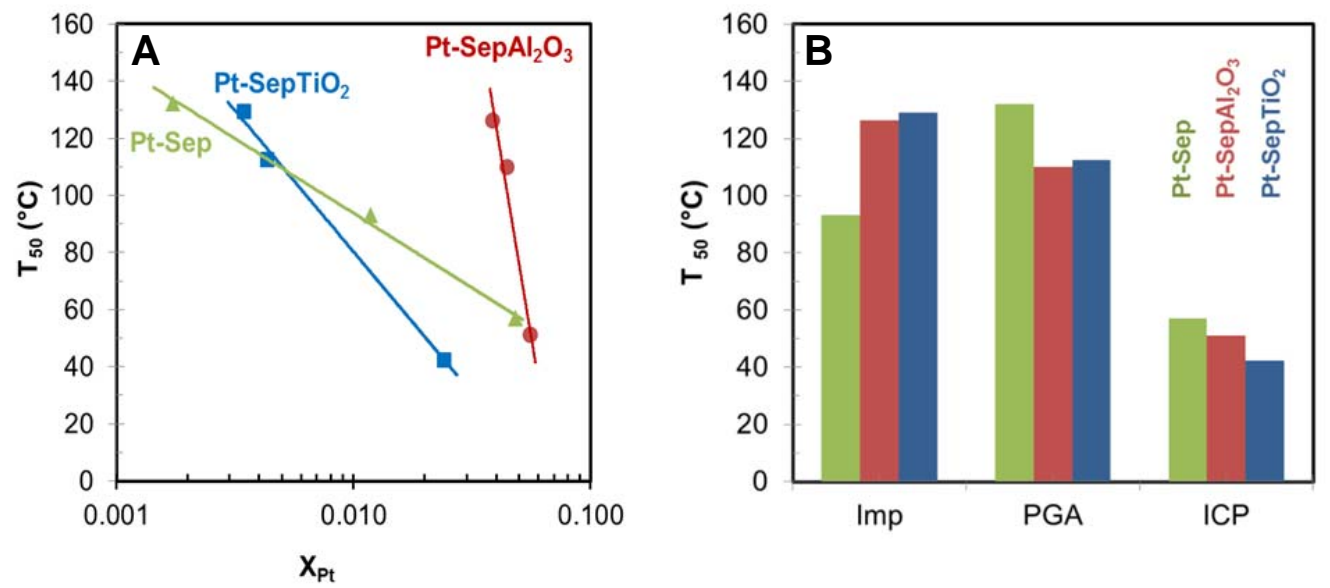

Figure 5. Temperature of VOCs half conversion for the three support compositions studied as a function of A) catalyst surface coverage, and B) preparation method.

Similar activity is obtained with different $\mathrm{Pt}$ dispersion depending on the support composition because the environment plays also a role in the properties of the active phase, modifying the acidity, the oxidation state or accessibility. Among ICP samples, Sep- $\mathrm{TiO}_{2}$ support leads to the lowest platinum apparent surface coverage, but the best activity. One possible reason might be the significantly higher interaction of $\mathrm{TiO}_{2}$ with the formaldehyde [36]. In metal oxide surfaces HCHO adsorption takes place via dioxymethylene formation. A lone pair donation from the oxygen of the carbonyl to Lewis acid sites, such as Ti cations, increases the electrophilicity of the carbonyl, favoring the nucleophilic attack from surface oxygen to the carbonyl [37]. Another reason may be the masking by sepiolite of surface titania particles, where the active phase is preferentially located, with effect in the $\mathrm{X}_{\mathrm{Pt}}$ estimation but not in the accessibility for the 
catalytic reaction due to the high porosity. It has previously been observed by the authors for $\mathrm{TiO}_{2}$ [35], $\mathrm{Al}_{2} \mathrm{O}_{3}[38]$ and also other oxides such as $\mathrm{VO}_{\mathrm{x}}-\mathrm{ZrO}_{2}-\mathrm{SO}_{4}{ }^{2-}$ [39], that in mixed materials sepiolite tends to cover the metal oxide particles. The effect would not be so significant for alumina particles, of larger size.

\subsection{Morphology}

Platinum relative dispersion in the catalysts has been investigated by transmission electron microscopy as well; Figure 6 shows representative micrographs. Sepiolite fibers are mainly free from $\mathrm{Pt}$ in the samples where macroporosity was created with AC, which is indicative of the aforementioned low affinity of the silicate for the active phase precursor.
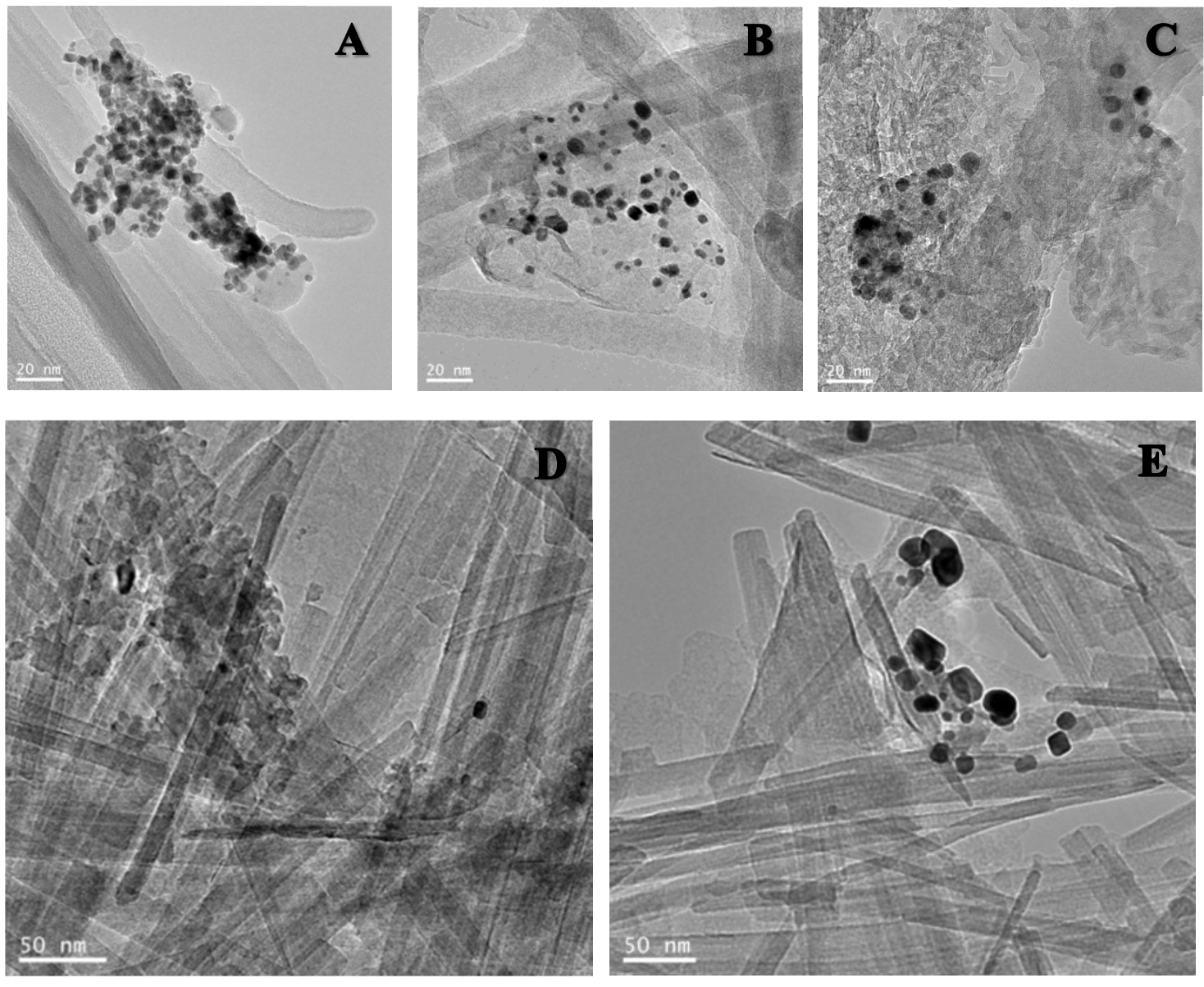

Figure 6. TEM micrographs of A) Pt-Sep (ICP), B) Pt-SepTiO 2 (ICP), C) Pt-SepAl $\mathrm{O}_{3}$ (ICP), D) Pt-Sep (IMP), and E) Pt-Sep (PGA). C and D micrographs have higher magnification.

The microscopic findings are in agreement with $\mathrm{X}_{\mathrm{Pt}}$ values. The smallest $\mathrm{Pt}$ particles are formed by ICP synthesis method $(<10 \mathrm{~nm}$, Figure 6A-C). The size of Pt particles observed by TEM varies in the sepiolite series from 3-5 nm in the ICP-catalyst (Figure 6A) to big particles of $20-40 \mathrm{~nm}$ in the PGA-catalyst (Figure 6E). As intermediate situation, Pt-Sep (Imp) has Pt particles of sizes between 5 and $20 \mathrm{~nm}$ (Figure 6D), similarly to both Imp- and PGA- catalysts of both Pt-SepAl $\mathrm{O}_{3}$ and Pt-SepTiO 2 series (not shown), where the noble metal is preferentially associated to the alumina or titania 
particles due to the positive charge of their surface. For this reason, despite the generation of big pores the noble metal does not aggregate so easily in the mixed supports.

\subsection{Phase distribution}

The tendency of the platinum anionic salt to avoid the sepiolite surface during the impregnation is confirmed analyzing the distribution of $\mathrm{Si}, \mathrm{Mg}, \mathrm{Al}, \mathrm{Ti}$ and $\mathrm{Pt}$ for each synthesis method in the micrographies obtained by WDS-EPMA shown in Figures 7 and 8.

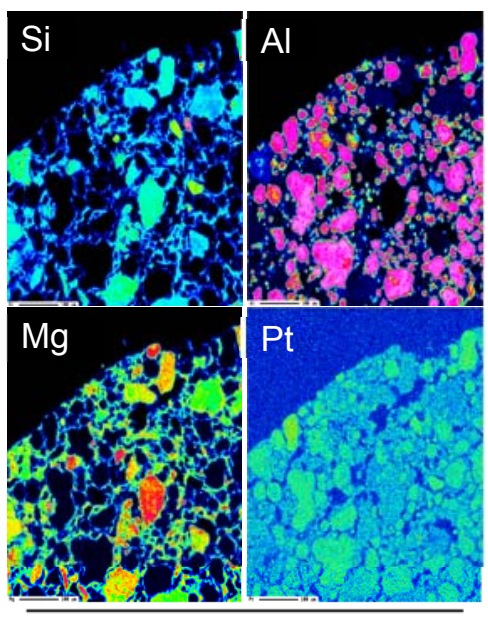

A) Pt-SepAl ${ }_{2} \mathrm{O}_{3}(\mathrm{Imp})$

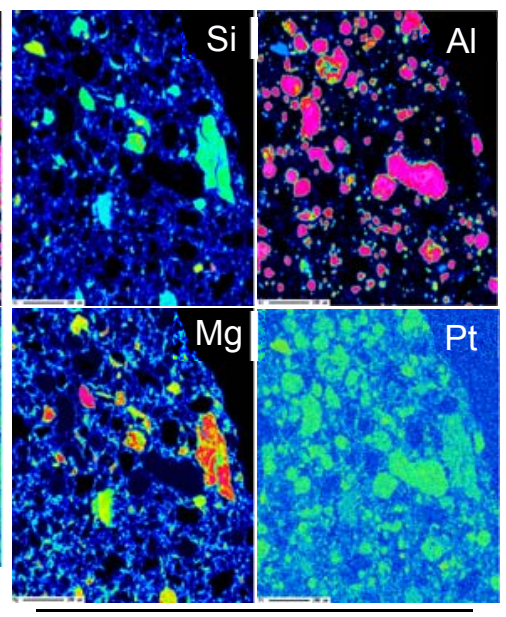

B) Pt-SepAl ${ }_{2} \mathrm{O}_{3}$ (PGA)

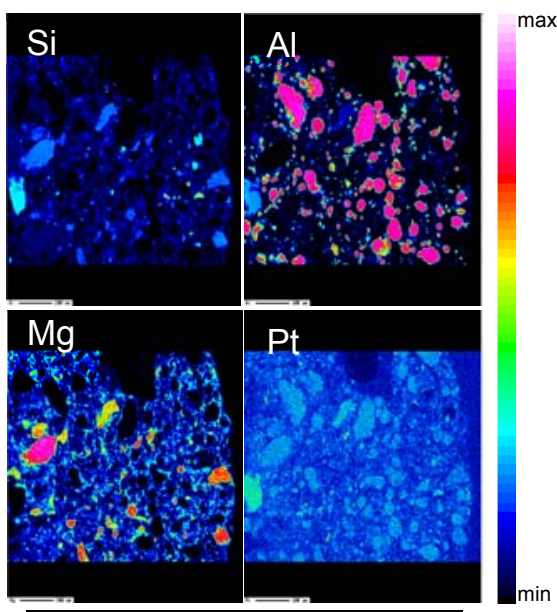

C) Pt-SepAl ${ }_{2} \mathrm{O}_{3}$ (ICP)

Figure 7.- EPMA mapping of the Pt-SepAl ${ }_{2} \mathrm{O}_{3}$ series.

$200 \mu \mathrm{m}$

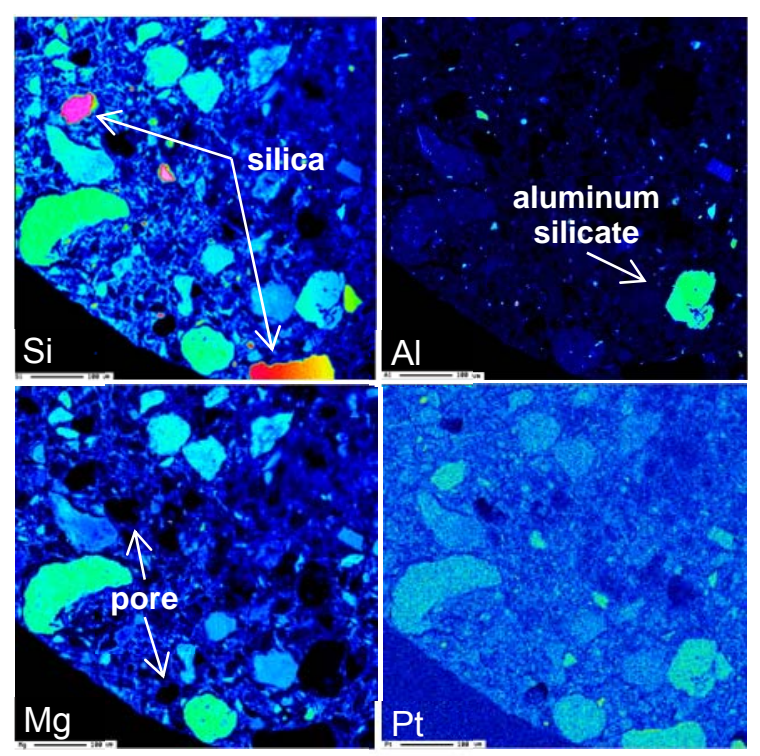

A) Pt-Sep (ICP)
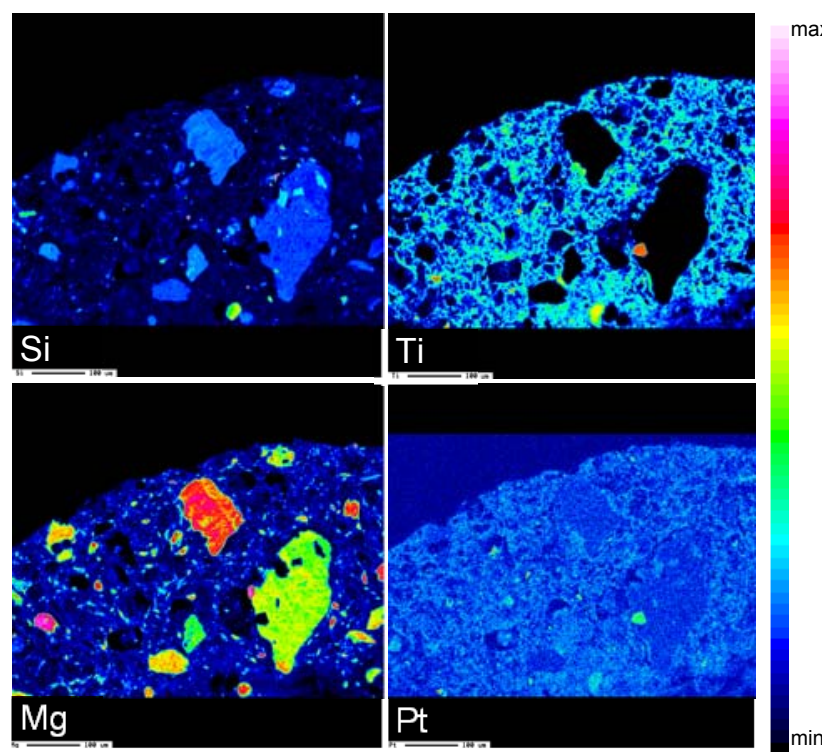

B) Pt-SepTiO (ICP)

Figure 8. EPMA mapping of A) Pt-Sep (ICP) and B) Pt-Sep $\mathrm{TiO}_{2}$ (ICP).

$200 \mu \mathrm{m}$

The pores are the areas that appear in black in all mappings. The higher porosity of Pt$\mathrm{SepAl}_{2} \mathrm{O}_{3}(\mathrm{PGA})$ and Pt-Sep $\mathrm{Al}_{2} \mathrm{O}_{3}(\mathrm{ICP})$, evident if Figure $7 \mathrm{~B}$ and $7 \mathrm{C}$ are compared to Figure 7A, is in agreement with MIP results (Table 2) and similar results are obtained in the equivalent preparations of the other two series (not shown). Alumina particles of the Pt-SepAl ${ }_{2} \mathrm{O}_{3}$ series are identified by $\mathrm{Al}$ rich areas coincident with black areas in $\mathrm{Si}$ and 
Mg mappings, indicative of the lack of both elements. Similarly, titania particles of the $\mathrm{Pt}-\mathrm{SepTiO}{ }_{2}$ series are identified by Ti rich areas. Magnesium rich areas, directly related to most silicon-rich areas, are indicative of the presence of sepiolite. Finally, the most significant impurities of the natural clay include aluminum silicate and quartz, which can be identified by the simultaneous presence of silicon and aluminum and the presence of only silicon, respectively. The mapping of platinum indicates that despite its low concentration $(0.2 \%)$ it is present in the entire catalyst particles. In Imp- and PGAcatalysts platinum is preferably associated with the metal oxide and aluminum silicate impurities (see Figures 7A and 7B for alumina series). These preference explains that the addition of a metal oxide support to sepiolite without PGA is detrimental for the catalytic activity, because the accessibility of the active phase is limited. However, in the case of the catalyst obtained by the ICP method platinum concentration is more homogenously distributed, with similar concentration in the magnesium silicate and the oxide (see Figure 7C for the alumina series and Figure $8 \mathrm{~B}$ for the titania series). The higher concentration of Pt coincident with sepiolite particles in ICP samples can be explained considering that in this case the incorporation of Pt onto the support surface does not occur in liquid phase. The platinum precursor is impregnated onto the activated carbon added to the dough. Therefore, while the latter is burned in a controlled oxygen atmosphere, a reducing atmosphere is generated due to $\mathrm{CO}$ formation and the platinum is reduced in the gas phase, being deposited on the closest sepiolite fibers to the carbon particles. Thus, the repulsion between the precursor ionic form $\left(\left[\mathrm{Cl}_{6} \mathrm{Pt}\right]^{2-}\right)$ and the negatively charged sepiolite surface is not produced, and the aggregation avoided. The $\mathrm{Pt}$ particles obtained have a reduced size $(3-5 \mathrm{~nm})$.

\section{CONCLUSIONS}

The generation of macropores in shaped catalytic supports helps to reduce internal diffusion limitations in heterogeneous catalytic reactions. However, subsequent impregnation and drying processes are also affected by the porous distribution, and thus the properties of precursor, binder and support will determine if a global beneficial effect is observed in the catalyst performance.

The low ZPC of sepiolite favors the formation of Pt aggregates in the impregnation of the natural silicate with chloroplatinic acid. Therefore the inclusion of activated carbon as hard template in the pre-extrusion bracket causes a significant catalyst activity decrease in the Pt-impregnated sepiolite supports related to the higher size of the Pt particles formed in the macropores. However, alumina and titania have a relatively high PZC and thus in mixed supports the anionic noble metal precursor is better dispersed and preferentially located on the metal oxide particles.

The impregnated carbon procedure facilitates the active phase dispersion, which combined with the porosity improvement gives rise to very active catalysts obtained by a simplified procedure. The use of activated carbon as both sacrificial template and impregnation medium not only provides the catalytic bodies with macroporosity, but also leads to a better Pt surface coverage, and at the same time the temporally formed $\mathrm{CO}$ inside the pores reduces the noble metal in-situ. Thus, the resulting catalysts have highly available active sites with minimal mass transfer limitations, a combination which makes possible the elimination of volatile organic compounds by deep oxidation at very moderate temperature $\left(40-80{ }^{\circ} \mathrm{C}\right)$ using a fairly low noble metal content $(0.2 \% \mathrm{Pt})$.

\section{Acknowledgements}


The authors wish to acknowledge the financial support received from the Spanish Ministry (MAP-IT, CTQ2011-25517), the Comunidad de Madrid (Alccones, S2013/MAE-2985) and the Chilean Fondecyt (1130749).

\section{References}

[1] P. Ávila, M. Montes, E.E. Miró, Monolithic reactors for environmental applications: A review on preparation technologies, Chem. Eng. J., 109 (2005) 11-36.

[2] R.M. Heck, S. Gulati, R.J. Farrauto, The application of monoliths for gas phase catalytic reactions, Chem. Eng. J., 82 (2001) 149-156.

[3] P. Colombo, C. Vakifahmetoglu, S. Costacurta, Fabrication of ceramic components with hierarchical porosity, J. Mater. Sci., 45 (2010) 5425-5455.

[4] Z.-Y. Yuan, T.-Z. Ren, A. Vantomme, B.-L. Su, Facile and Generalized Preparation of Hierarchically Mesoporous-Macroporous Binary Metal Oxide Materials, Chem. Mater., 16 (2004) 5096-5106.

[5] V. Valtchev, Preparation of regular macroporous structures built of intergrown silicalite-1 nanocrystals, J. Mater. Chem., 12 (2002) 1914-1918.

[6] M. Schwickardi, T. Johann, W. Schmidt, F. Schüth, High-Surface-Area Oxides Obtained by an Activated Carbon Route, Chem. Mater., 14 (2002) 3913-3919.

[7] T. Yokota, Y. Takahata, T. Katsuyama, Y. Matsuda, A new technique for preparing ceramics for catalyst support exhibiting high porosity and high heat resistance, Catal. Today, 69 (2001) 11-15.

[8] O. Majoulet, F. Sandra, M.C. Bechelany, G. Bonnefont, G. Fantozzi, L. Joly-Pottuz, A. Malchere, S. Bernard, P. Miele, Silicon-boron-carbon-nitrogen monoliths with high, interconnected and hierarchical porosity, J. Mater. Chem. A, 1 (2013) 10991-11000.

[9] J.H. She, T. Ohji, S. Kanzaki, Oxidation bonding of porous silicon carbide ceramics with synergistic performance, J. Eur. Ceram. Soc., 24 (2004) 331-334.

[10] G. Hasegawa, K. Kanamori, K. Nakanishi, T. Hanada, Facile preparation of hierarchically porous TiO2 monoliths, J. Am. Ceram. Soc., 93 (2010) 3110-3115.

[11] X. Guo, W. Li, K. Nakanishi, K. Kanamori, Y. Zhu, H. Yang, Preparation of mullite monoliths with well-defined macropores and mesostructured skeletons via the sol-gel process accompanied by phase separation, J. Eur. Ceram. Soc., 33 (2013) 1967-1974.

[12] X. Guo, K. Nakanishi, K. Kanamori, Y. Zhu, H. Yang, Preparation of macroporous cordierite monoliths via the sol-gel process accompanied by phase separation, J. Eur. Ceram. Soc., (2013).

[13] S. Suárez, M. Yates, P. Avila, J. Blanco, New TiO2 monolithic supports based on the improvement of the porosity, Catal. Today, 105 (2005) 499-506.

[14] J. Blanco, A.L. Petre, M. Yates, M.P. Martin, S. Suarez, J.A. Martin, Novel One-Step Synthesis of Porous-Supported Catalysts by Activated-Carbon Templating, Advanced Materials, 18 (2006) 1162-1165.

[15] A.J. Blanco, G.P. Ávila, L.C. Chacón, J.J.M. Ramos, Method for the preparation of metal or metal oxide catalysts that are supported on porous materials, EP1952885A1 (2008).

[16] P.T. Anastas, J.C. Warner, Green Chemistry: Theory and Practice, Oxford University Press, New York, 1998.

[17] J. Blanco, A.L. Petre, M. Yates, M.P. Martin, J.A. Martin, M.A. Martin-Luengo, Tailormade high porosity VOC oxidation catalysts prepared by a single-step procedure, Appl. Catal., B, 73 (2007) 128-134.

[18] P. Avila, J. Blanco, C. Knapp, M. Yates, Platinum-titania-sepiolite monolithic catalysts for the reduction of nitric oxide with propene in lean-burn conditions, Stud. Surf. Sci. Catal., 1998, pp. 233-242.

[19] S. Suárez, M. Yates, A.L. Petre, J.A. Martín, P. Avila, J. Blanco, Development of a new $\mathrm{Rh} / \mathrm{TiO} 2-$ sepiolite monolithic catalyst for $\mathrm{N} 2 \mathrm{O}$ decomposition, Appl. Catal., B, 64 (2006) 302-311. 
[20] E.W. Washburn, The Dynamics of Capillary Flow, Physical Review, 17 (1921) 273283.

[21] J. Rouquerol, D. Avnir, C.W. Fairbridge, D.H. Everett, J.M. Haynes, N. Pernicone, J.D.F. Ramsay, K.S.W. Sing, K.K. Unger, Recommendations for the characterization of porous solids (Technical Report), Pure Appl. Chem., 66 (1994).

[22] E.P. Barrett, L.G. Joyner, P.P. Halenda, The Determination of Pore Volume and Area Distributions in Porous Substances. I. Computations from Nitrogen Isotherms, J. Am. Chem. Soc., 73 (1951) 373-380.

[23] M. Villarroel, P. Baeza, F. Gracia, N. Escalona, P. Avila, F.J. Gil-Llambías, Phosphorus effect on $\mathrm{Co} / \mathrm{Mo}$ and $\mathrm{Ni} / / \mathrm{Mo}$ synergism in hydrodesulphurization catalysts, Appl. Catal., A, 364 (2009) 75-79.

[24] P. Avila, J. Blanco, A. Bahamonde, J.M. Palacios, C. Barthelemy, Influence of the binder on the properties of catalysts based on titanium-vanadium oxides, J. Mater. Sci., 28 (1993) 4113-4118.

[25] J. Blanco, M. Yates, P. Avila, A. Bahamonde, Characterization of alumina:sepiolite monoliths for use as industrial catalyst supports, J. Mater. Sci., 29 (1994) 5927-5933.

[26] A. Álvarez, Sepiolite: properties and uses, in: A. Singer, E. Galán (Eds.) Palygorskite-sepiolite occurrences, genesis, and uses Elsevier, Amsterdam, 1984, pp. 253-289.

[27] F.E. Tuler, R. Portela, P. Ávila, E.D. Banús, E.E. Miró, V.G. Milt, Structured catalysts based on sepiolite with tailored porosity to remove diesel soot, Appl. Catal., A, 498 (2015) 41-53.

[28] S. Suárez, J.M. Coronado, R. Portela, J.C. Martín, M. Yates, P. Ávila, B. Sánchez, On the preparation of TiO2-sepiolite hybrid materials for the photocatalytic degradation of TCE: influence of TiO2 distribution in the mineralization., Environ. Sci. Technol., 42 (2008) 5892-5896.

[29] E. Ruiz-Hitzky, Molecular access to intracrystalline tunnels of sepiolite, J. Mater. Chem., 11 (2001) 86-91.

[30] R. Portela, F. Rubio-Marcos, P. Leret, J.F. Fernandez, M.A. Banares, P. Avila, Nanostructured $\mathrm{ZnO} /$ sepiolite monolithic sorbents for $\mathrm{H} 2 \mathrm{~S}$ removal, J. Mater. Chem. A, 3 (2015) 1306-1316.

[31] G.A. Parks, The Isoelectric Points of Solid Oxides, Solid Hydroxides, and Aqueous Hydroxo Complex Systems, Chemical Reviews, 65 (1965) 177-198.

[32] F.J. Gil-Llambias, A.M. Escudey-Castro, Use of zero point charge measurements in determining the apparent surface coverage of molybdena in $\mathrm{MoO} 3 /[\mathrm{gamma}-\mathrm{Al} 2 \mathrm{O} 3$ catalysts, J. Chem. Soc., Chem. Commun., (1982) 478-479.

[33] C. Perego, P. Villa, Catalyst preparation methods, Catal. Today, 34 (1997) 281-305. [34] G.H. Van Den Berg, H.T. Rijnten, The Impregnation and Drying Step in Catalyst Manufacturing, in: P.G.P.J. B. Delmon, G. Poncelet (Eds.) Stud. Surf. Sci. Catal., Elsevier1979, pp. 265-277.

[35] C. Knapp, F.J. Gil-Llambias, M. Gulppi-Cabra, P. Avila, J. Blanco, Phase distribution in titania-sepiolite catalyst supports prepared by different methods, J. Mater. Chem., 7 (1997) 1641-1645.

[36] R. Portela, I. Jansson, S. Suárez, M. Villarroel, B. Sánchez, P. Ávila, Insights on the adsorptive-photocatalytic properties of natural silicate-TiO2 hybrids for formaldehyde removal in air, submitted.

[37] D. Chen, Z. Qu, Y. Sun, K. Gao, Y. Wang, Identification of reaction intermediates and mechanism responsible for highly active $\mathrm{HCHO}$ oxidation on $\mathrm{Ag} / \mathrm{MCM}-41$ catalysts, Appl. Catal., B, 142-143 (2013) 838-848.

[38] S. Suárez, C. Saiz, M. Yates, J.A. Martin, P. Avila, J. Blanco, Rh/Y-Al2O3-sepiolite monolithic catalysts for decomposition of N2O traces, Appl. Catal., B, 55 (2005) 57-64.

[39] S.B. Rasmussen, J. Due-Hansen, M. Villarroel, F.J. Gil-Llambias, R. Fehrmann, P. Ávila, Multidisciplinary determination of the phase distribution for VO X-ZrO 2-SO 4 2-sepiolite catalysts for NH 3-SCR, Catal. Today, 172 (2011) 73-77. 